\title{
Magnetic resonance imaging features of the biceps tendon rupture in a Labrador retriever dog
}

\author{
Hyunju Cho, Hojung Choi, Youngwon Lee* \\ College of Veterinary Medicine and Research Institute of Veterinary Medicine, Chungnam National University, \\ Daejeon 34134, Korea
}

\begin{abstract}
A five-year-old spayed female Labrador retriever presented with right forelimb lameness one day earlier. Radiographs and computed tomography of the right shoulder joint revealed conspicuous enthesophytes, bone cyst, and bone fragment at the intertubercular groove. Magnetic resonance imaging showed a loss of continuity of the biceps tendon and inhomogeneous hyperintense signal of the surrounding soft tissue and moderate synovial fluid on T2- and proton density-weighted images with contrast enhancement on the postcontrast T1-weighted images. The dog was diagnosed with tenosynovitis and biceps tendon rupture with osteoarthritis based on a comprehensive evaluation of the computed tomography and magnetic resonance imaging features.
\end{abstract}

Keywords: shoulder joint disease, CT, MRI, dog, biceps tendon rupture

*Corresponding author

Youngwon Lee

College of Veterinary Medicine and Research Institute of Veterinary Medicine, Chungnam

National University, 99 Daehak-ro, Yuseonggu, Daejeon 34134, Korea

Tel: $+82-821-6786$

Fax: $+82-821-6703$

E-mail: lywon@cnu.ac.kr

ORCID

Hyungju Cho

https://orcid.org/0000-0003-0967-1890

Hojung Choi

https://orcid.org/0000-0001-7167-0755

Youngwon Lee

https://orcid.org/0000-0003-3207-0989

Conflict of Interest

The authors declare no conflicts of interest.

Received: July 24, 2020

Revised: October 8, 2020

Accepted: October 12, 2020
A five-year-old, spayed female Labrador retriever dog weighing $25 \mathrm{~kg}$ was referred to that authors with a history of acute onset right forelimb weightbearing lameness in a swimming pool one day before presentation. The physical examination revealed severe pain response when operating the right shoulder joint without joint swelling. The complete blood count was within the normal reference range. The C-reactive protein increased above the normal range $(8.1 \mathrm{mg} / \mathrm{L}$, reference range: $0-2 \mathrm{mg} / \mathrm{L})$ in serum biochemistry.

Radiographs of the right shoulder joint revealed sclerosis of the intertubercular groove, enthesophyte formation on the supra-/infra-glenoid tubercle, osteophyte of the humeral head, and widening of the right glenohumeral joint space (Fig. 1A). No significant findings were found on the left shoulder joint. A computed tomography (CT) examination was planned for an accurate evaluation of bone lesions.

The dog was sedated with midazolam $(0.2 \mathrm{mg} / \mathrm{kg}, \mathrm{IV})$ and anesthetized with propofol (4 mg/kg, IV). After intubation, anesthesia was maintained with isoflurane in oxygen.

A CT examination was performed using a helical CT scanner (Alexion, Canon, Japan), and the following parameters were used: $120 \mathrm{kV}, 150 \mathrm{mAs}, 1$ $\mathrm{mm}$ slice thickness, 0.75 second rotation time, and a 0.938 collimation beam pitch with a bone algorithm (window width: 2,700, window level: 350 ). The bilateral shoulder joints were scanned in the dorsal plane with lying on the left side with the patient's forelimbs pulled forward. Transverse and sagittal plane images were reconstructed for a three-dimensional evaluation. CT confirmed the prominent enthesophytes in the scapular glenoid cavity and humeral head, bone fragment located on the intertubercular groove, and subchondral bone cysts in the greater tubercle of the right shoulder joint. In particular, the intertubercular groove and the insertion site of the biceps tendon had a very irregular margin due to the formation of enthesophytes (Fig. 1B-E). The radiographs and CT images indicated biceps tenosynovitis with degenerative joint disease. As acute lameness occurred, however, tendon and ligament damage was suspected given the history of the slippery swimming pool. Therefore, additional magnetic resonance imaging (MRI) examination was conducted to check for soft tissue injury.

MRI was performed using a 1.5 Tesla magnet scanner (Vantage Elan, 


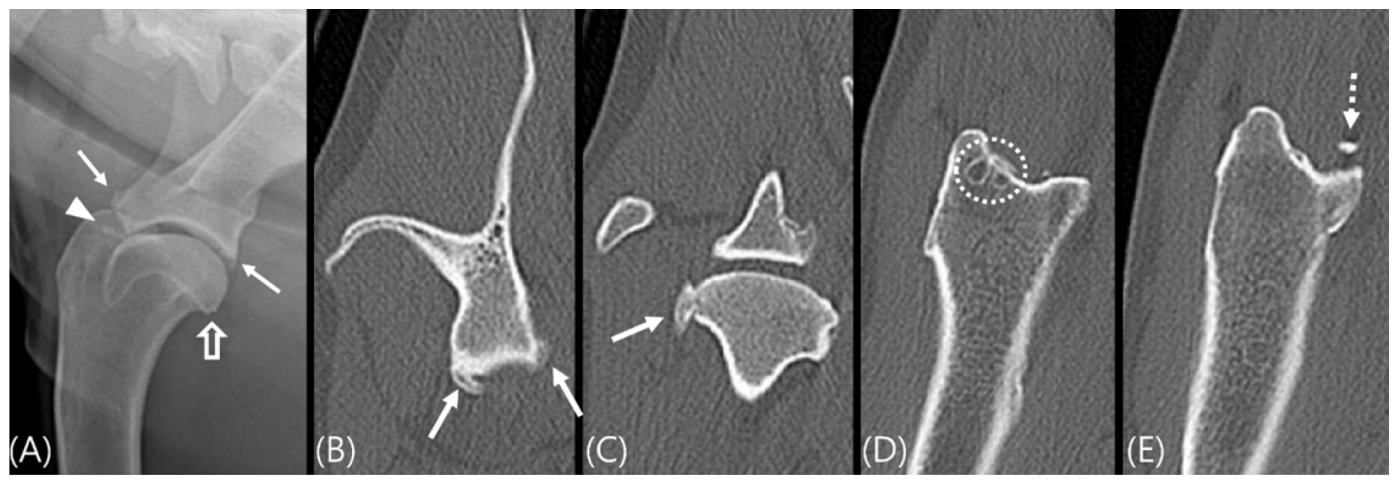

Fig. 1. Lateral radiograph (A) and dorsal CT images at the glenoid cavity level (B), humeral head level (C), and intertubercular groove level (D, E) of right shoulder joint. On the radiograph (A), note the sclerosis of the intertubercular groove (arrowhead), enthesophyte formation on the supra/infra-glenoid tubercle (arrows), osteophyte of the humeral head (open arrow), and widening of the right glenohumeral joint. CT examination revealed not only enthesophyte in the glenoid cavity and humeral head (arrows, B, C) but also bone cysts (dotted circle) and calcifying body (dashed arrow). CT, computed tomography.

Canon). In addition to the spine coil, a 16 channel flexible coil was used to improve image quality. To cover it close to the shoulder, the patient was positioned in lateral recumbency with the injured shoulder upward. The images were obtained using spin-echo sequences. T1-weighted images (T1WI) $(\mathrm{TR}=600 \mathrm{~ms}$ and $\mathrm{TE}=10 \mathrm{~ms}$, matrix size $=224 \times$ 256), T2-weighted images (T2WI) $(\mathrm{TR}=3,200 \mathrm{~ms}$ and $\mathrm{TE}=$ $70 \mathrm{~ms}$, matrix size $=192 \times 224)$, proton density-weighted images $(\mathrm{PDWI})(\mathrm{TR}=1,900 \mathrm{~ms}$ and $\mathrm{TE}=15 \mathrm{~ms}$, matrix size $=192 \times 240)$ and PDW with fat saturation (PDW fat sat) images $(\mathrm{TR}=2,100 \mathrm{~ms}$ and $\mathrm{TE}=15 \mathrm{~ms}$, matrix size $=192 \times$ 192) of the bilateral shoulder joint were obtained. All sequences were scanned in the transverse, sagittal, and dorsal plane with a $13 \times 13 \mathrm{~cm}$ field of view, $3.0 \mathrm{~mm}$ slice thickness, and $0.3 \mathrm{~mm}$ inter-slice gap. Gadodiamide at a dose of $0.3 \mathrm{~mL} / \mathrm{kg}$ (Omniscan, Nycomed Imaging AS, Norway) was injected intravenously by hand, and T1W SE was repeated immediately. The digital imaging and communications in medicine files for each shoulder MRI study were retrieved and analyzed on a computer workstation.

On MRI, the right biceps tendon revealed a loss of continuity in the intertubercular groove with the moderate right glenohumeral joint effusion. This tendon showed hyperintense signal and heterogeneous parenchyma on the $\mathrm{T} 2 \mathrm{~W}$ and PDW images (Fig. 2A-D). In addition, the medial glenohumeral ligament and subscapularis tendon was thickened (Fig. $2 \mathrm{E}$ and $\mathrm{F}$ ). Contrast-enhanced T1W images showed heterogeneous enhancement in the overall right shoulder joint capsule (Fig. 3).

All findings observed in the CT and MR images were confirmed visually by arthroscopy. Biceps tendon rupture and partial rupture of both the medial glenohumeral ligament and subscapularis tendon of the right shoulder joint were confirmed visually. After removing the bone fragment around the biceps tendon, later monitoring showed improvement of the lameness.

The canine shoulder is a complex joint with numerous pas- sive and active soft tissue stabilizing components. Therefore, determining the exact causes of shoulder lameness is often challenging. Preoperative diagnostic tests, such as refined physical examination, radiographs, standard arthrography, ultrasound, CT, MRI, and MR arthrography, were performed [1]. Standard radiographs and arthrography provide only limited diagnostic information [2]. Ultrasonography is useful for providing localized views of superficial structures of the shoulder, but an evaluation of the deeper soft tissue structures is limited by bone interference [3]. CT allows better visualization of osseous structures than conventional radiography by eliminating the opacities caused by superimposed tissue. In addition, the ability to reconstruct images in any image plane can help evaluate complex fractures and articular trauma. On the other hand, precise identification of the soft tissue structures with similar densities remains difficult [4]. MRI is considered a powerful diagnostic modality because it can evaluate the internal and external structures simultaneously as high soft-tissue contrast, high resolution, and multi-plane imaging capabilities [1,5-7].

An MRI examination revealed inflammation of the biceps tendon appeared as an enlargement tendon with heterogeneous signal intensity on T2WI, T1WI, and PDWI, and as an increase in signal intensity on the T2WI and PDWI [1]. The hyperintense linear signal across the tendon on the T2W and PDW images is also an accurate sign of tendon rupture with a morphologic change. Tendon retraction, muscle atrophy, and fatty infiltration are important prognostic factors. Distension of the shoulder joint capsule and accumulation of T2 hyperintense fluid are commonly seen because the tendon sheath communicates with the synovial joint. In chronic cases, thickening of the synovium due to synovitis is observed as a hypointense thick lining on T2WI with strong enhancement on postcontrast T1WI $[8,9]$.

Of the various MRI sequences, T2W and PDW images were good for assessing the parenchymal changes. The PDW fat sat and $\mathrm{T} 2 \mathrm{~W}$ images were most efficient in identifying 


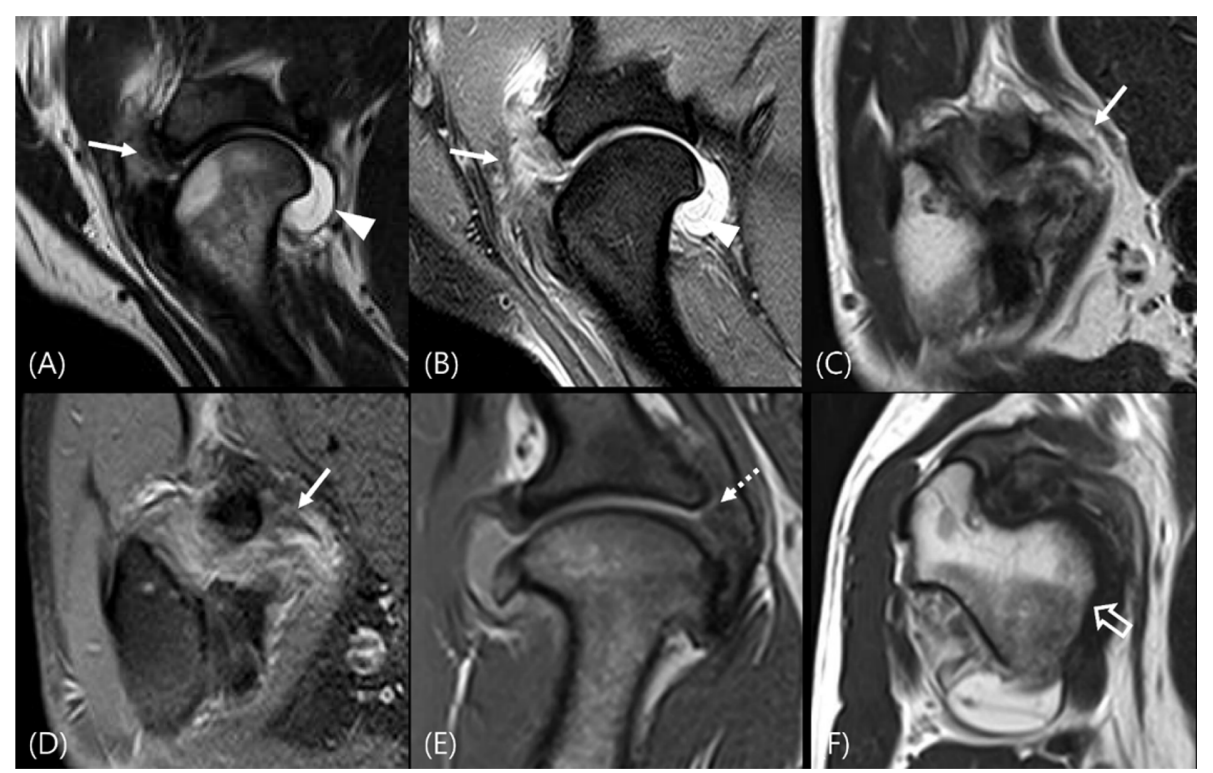

Fig. 2. Sagittal T2W (A), sagittal PDW (B), dorsal T2W (C), dorsal PDW at the biceps tendon level (D), dorsal PDW at the medial glenohumeral ligament level (E), and transverse $\mathrm{T} 2 \mathrm{~W}$ at the subscapularis tendon $(\mathrm{F})$ magnetic resonance images of the right shoulder joint. There are the loss of continuity of biceps tendon and heterogeneous hyperintensity around it (arrows, A-D) with the moderate right glenohumeral synovial fluid (arrowhead, A, B). In addition, thickening of the medial glenohumeral ligament (dashed arrow, E) and subscapularis tendon (open arrow, F) were revealed. T2W, T2-weighted; PDW, proton density-weighted.

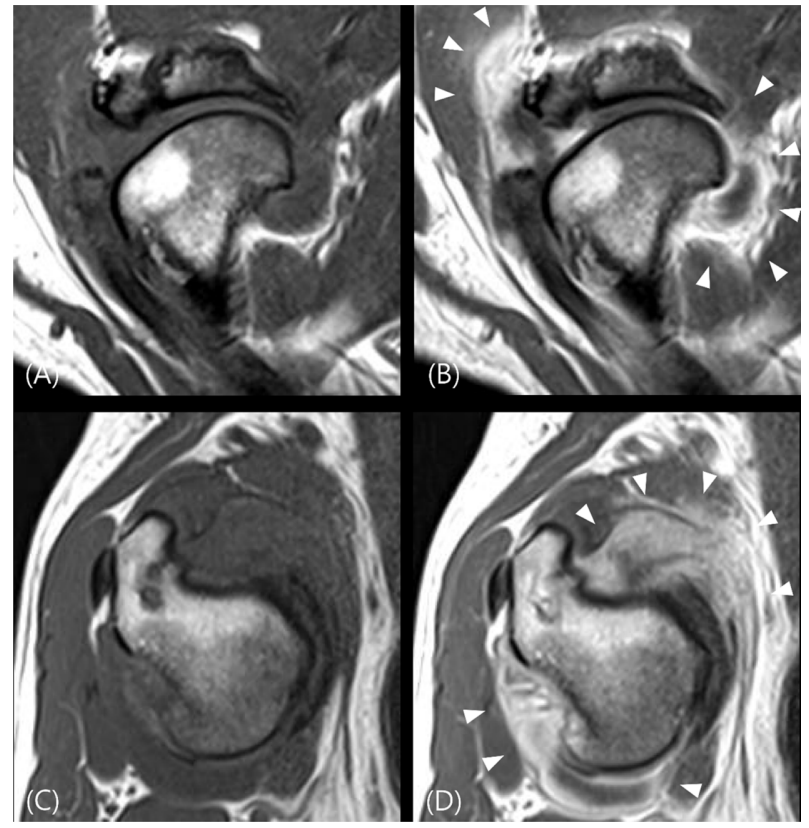

Fig. 3. Sagittal (A) and transverse (C) T1-weighted pre-contrast, and sagittal (B) and transverse (D) postcontrast magnetic resonance images of the right shoulder joint. There was strong heterogeneous contrast enhancement (arrowheads) of the right shoulder joint capsule.

fluid accumulation in the joint and around the tendons. In addition, postcontrast $\mathrm{T} 1 \mathrm{~W}$ images confirmed the overall enhancement effect of the joint capsule. This was useful for identifying secondary inflammatory changes in the synovial membrane.

In a human medical study, MRI and arthrography had a similar diagnostic and therapeutic impact and comparable accuracy. The clinician could make a more confident diagnosis after either one of these imaging tests. A certain diagnosis could be made on only one of the radiological techniques. For example, tendinitis was not an arthrographic diagnosis but was frequently diagnosed on MRI; conversely, capsulitis was only commented at arthrography. In one study, when MRI and arthrography were applied randomly, both tests led to a similar number of new diagnoses that were not previously considered in $24-34 \%$ of the patients examined, such as acromioclavicular joint degeneration, capsulitis, and impingement $[10,11]$. Because of the effectiveness evaluation of the shoulder MRI, MRI altered the primary diagnosis in $23-68 \%$ of cases, and management plans were subsequently modified in $15-61 \%$ of cases [12].

In veterinary medicine, one study evaluated the diagnostic potential of shoulder MRI compared to a reference standard, arthroscopy, and open surgery [1]. In all ligament or tendon diseases, on average, the results showed $90 \%$ agreement and $87 \%$ concordance for each assessment. In this case, however, partial ruptures of the medial glenohumeral ligament and subscapularis tendon were not observed and only appeared as thickening on the MR images. An analysis of the MR diagnosis of partial tears in human medicine revealed MRI to be less satisfactory [13]. There are two main causes of this: anatomical position and image quality. On the coro- 
nal images, subtle partial tears of subscapularis tendon may not be properly assessed because the subscapularis muscle lies in the same anatomic plane. Hence, axial plane images are essential for detecting subscapularis tendon injuries and associated lesions of the biceps tendon [3]. Another reason for the false-negative findings is the partial volume effect. Small tears in this region can be particularly difficult to observe because of the blurred surfaces of the structures [14]. To overcome these disadvantages, angled images with smaller section thickness and interslice gap and fat sat technique can be applied. The angled images will have a less partial volume effect because of the smaller voxel size and less unimaged tendon in the interslice gap. Fat sat images increase the visibility of water on T2WI and reduce the signal from the adipose tissue that might confound the image interpretation $[6,14,15]$.

In conclusion, this report described the $\mathrm{CT}$ and MR findings of the biceps tendon rupture. As CT and MRI have different advantages, a comprehensive evaluation is needed when the patient has forelimb lameness. In particular, MR imaging is very useful for a diagnosis of soft tissue injury.

\section{References}

1. Innes JF, Brown G. Rupture of the biceps brachii tendon sheath in two dogs. J Small Anim Pract 2004;45:25-28.

2. Bruce WJ, Spence S, Miller A. Teres minor myopathy as a cause of lameness in a dog. J Small Anim Pract 1997;38:7477.

3. Deutsch A, Altchek DW, Veltri DM, Potter HG, Warren RF. Traumatic tears of the subscapularis tendon. Clinical diagnosis, magnetic resonance imaging findings, and operative treatment. Am J Sports Med 1997;25:13-22.

4. Hoskinson JJ, Tucker RL. Diagnostic imaging of lameness in small animals. Vet Clin North Am Small Anim Pract 2001; $31: 165-180$.
5. Kunkel KA, Rochat MC. A review of lameness attributable to the shoulder in the dog: part two. J Am Anim Hosp Assoc 2008:44:163-170.

6. Mirowitz SA. Normal rotator cuff: MR imaging with conventional and fat-suppression techniques. Radiology 1991; 180:735-740

7. Murphy SE, Ballegeer EA, Forrest LJ, Schaefer SL. Magnetic resonance imaging findings in dogs with confirmed shoulder pathology. Vet Surg 2008;37:631-638.

8. Sage JE, Gavin P. Musculoskeletal MRI. Vet Clin North Am Small Anim Pract 2016;46:421-451.

9. Shahabpour M, Kichouh M, Laridon E, Gielen JL, De Mey J. The effectiveness of diagnostic imaging methods for the assessment of soft tissue and articular disorders of the shoulder and elbow. Eur J Radiol 2008;65:194-200.

10. Blanchard TK, Mackenzie R, Bearcroft PW, Sinnatamby R, Gray A, Lomas DJ, Constant CR, Dixon AK. Magnetic resonance imaging of the shoulder: assessment of effectiveness. Clin Radiol 1997;52:363-368.

11. Blanchard TK, Bearcroft PW, Maibaum A, Hazelman BL, Sharma S, Dixon AK. Magnetic resonance imaging or arthrography for shoulder problems: a randomised study. Eur J Radiol 1999;30:5-10.

12. Bearcroft PW, Blanchard TK, Dixon AK, Constant CR. An assessment of the effectiveness of magnetic resonance imaging of the shoulder: literature review. Skeletal Radiol 2000;29:673-679.

13. Iannotti JP, Zlatkin MB, Esterhai JL, Kressel HY, Dalinka MK, Spindler KP. Magnetic resonance imaging of the shoulder. Sensitivity, specificity, and predictive value. J Bone Joint Surg Am 1991;73:17-29.

14. Tuite MJ, Asinger D, Orwin JF. Angled oblique sagittal MR imaging of rotator cuff tears: comparison with standard oblique sagittal images. Skeletal Radiol 2001;30:262-269.

15. Patten RM, Spear RP, Richardson ML. Diagnostic performance of magnetic resonance imaging for the diagnosis of rotator cuff tears using supplemental images in the oblique sagittal plane. Invest Radiol 1994;29:87-93. 